\title{
THE COMPLEXITY OF THE EXTENDIBILITY PROBLEM FOR FINITE POSETS*
}

\author{
BENOIT LAROSE ${ }^{\dagger}$ AND LÁSZLÓ ZÁDORI ${ }^{\ddagger}$
}

\begin{abstract}
For a finite poset $P$ let $\operatorname{EXT}(P)$ denote the following decision problem. Given a finite poset $Q$ and a partial map $f$ from $Q$ to $P$, decide whether $f$ extends to a monotone total map from $Q$ to $P$.

It is easy to see that $\operatorname{EXT}(P)$ is in the complexity class NP. In [SIAM J. Comput., 28 (1998), pp. 57-104], Feder and Vardi define the classes of width 1 and of bounded strict width constraint satisfaction problems for finite relational structures. Both classes belong to the broader class of bounded width problems in $\mathbf{P}$. We prove that for any finite poset $P$, if $\operatorname{EXT}(P)$ has bounded strict width, then it has width 1 . In other words, if a poset admits a near unanimity operation, it also admits a totally symmetric idempotent operation of any arity. In [Fund. Inform., 28 (1996), pp. 165-182], Pratt and Tiuryn proved that $\operatorname{SAT}(P)$, a polynomial-time equivalent of $\operatorname{EXT}(P)$ is NPcomplete if $P$ is a crown. We generalize Pratt and Tiuryn's result on crowns by proving that $\operatorname{EXT}(P)$, is NP-complete for any finite poset $P$ which admits no nontrivial idempotent Malcev condition.
\end{abstract}

Key words. NP-complete, extendibility problem for posets, constraint satisfaction problem, zigzags, totally symmetric idempotent and near unanimity operations

AMS subject classifications. 06A07, 68A20, 03C05

DOI. $10.1137 / \mathrm{S} 0895480101389478$

1. Introduction. We consider the following decision problem: Let $P$ and $Q$ be two finite posets and $f$ a partial map from $Q$ to $P$. Does $f$ extend to a monotone total map from $Q$ to $P$ ?

We get different problems depending on which of $P$ and $Q$ is considered as an input. As easily observed, the problem obtained in each case is contained in complexity class NP. The case when the problem takes $P, Q$, and $f$ as inputs is NP-complete, as was proved by Duffus and Goddard in [5]. For a fixed finite poset $P$ let $\operatorname{EXT}(P)$, denote the above problem with inputs $Q$ and $f$. We call $\operatorname{EXT}(P)$ the extendibility problem for $P$. The complexity of a decision problem called $\operatorname{SAT}(P)$, a polynomialtime equivalent of $\operatorname{EXT}(P)$, was studied by Pratt and Tiuryn in [13]. They proved that if $P$ is a crown, then $\operatorname{SAT}(P)$ is $\mathbf{N P}$-complete and also gave examples of $P$ when $\operatorname{SAT}(P)$ is in $\mathbf{P}$.

The height of a finite poset $P$ is defined to be the maximum value of $k$ such that there exists a $(k+1)$-element chain in $P$. A finite poset is dismantlable whenever its elements can be listed in such a way that each element, except the last one, has a unique upper or lower cover in the subposet determined by it and its successors. A connected finite poset is ramified if it has at least two elements and no element with a unique upper or lower cover.

A relational set (or structure) is defined here to be a set equipped with finitary relations. A relational set always is of a certain type and it is finite if its base set is

*Received by the editors April 20, 2001; accepted for publication (in revised form) May 7, 2003; published electronically October 2, 2003.

http://www.siam.org/journals/sidma/17-1/38947.html

${ }^{\dagger}$ Department of Mathematics and Statistics, Concordia University, 1455 de Maisonneuve West, Montreal, QC, Canada, H3G 1M8 (larose@mathstat.concordia.ca). The research of this author was supported by the Natural Sciences and Engineering Research Council of Canada.

${ }^{\ddagger}$ Bolyai Intézet, Aradi vértanúk tere 1, H-6720 Szeged, Hungary (zadori@math.u-szeged.hu). The research of this author was partially supported by OTKA grants T022867, T026243 and by FKFP grant 1259/1997. 
finite. A reduct of a relational set $R$ is a relational set obtained from $R$ by leaving out some relations. For a finite relational set $R$ we define a decision problem denoted by $\operatorname{CSP}(R)$ that is called the constraint satisfaction problem for $R$. An instance of $\operatorname{CSP}(R)$ is a finite-type reduct $R^{\prime}$ of $R$ and a finite relational set $X$ similar to $R^{\prime}$, and the question is whether there is a morphism from $X$ to $R^{\prime}$.

In the case when $R$ is of finite type, each instance $I$ related to $R^{\prime}$ is considered as an instance related to $R$ by supplying $I$ with additional empty relations, one for each relational symbol missing in the type of $R^{\prime}$. So if $R$ is of finite type, we conceive $\operatorname{CSP}(R)$ as a decision problem whose instances are the finite structures similar to $R$, and the question for an instance $I$ is if there is a homomorphism from $I$ to $R$. This point of view is followed by Feder and Vardi in [6] and makes only constant time difference in complexity compared with the general definition given for $\operatorname{CSP}(R)$ in the preceding paragraph.

In [6] Feder and Vardi proved that for every relational set $R$ of finite type $\operatorname{CSP}(R)$ is polynomial-time equivalent to the retraction problem for some finite poset $P$. The latter problem is easily shown to be equivalent to $\operatorname{SAT}(P)$; see [13]. It also turned out in [6] that $P$ might be assumed to be of height at most 2 and ramified. We mention that, in contrast with this result, Pratt and Tiuryn proved in [13] that for every poset $P$ of height $1, \operatorname{SAT}(P)$ is NP-complete if $P$ contains a crown and is in $\mathbf{P}$ otherwise. It is clear from the above result of Feder and Vardi that statements on the complexity of the extendibility problem for posets might have a bearing on the complexity of CS problems.

In [6] Feder and Vardi defined the notions of width and strict width for CS problems. They showed that CS problems of bounded width have polynomial-time complexity. CS problems of strict width $k$ are certain problems of width $k$. In [6] it was proved that $\operatorname{CSP}(R)$ has bounded strict width if and only if $R$ admits a near unanimity operation. It was also shown that $\operatorname{CSP}(R)$ has width 1 if and only if there is a certain structure that admits a homomorphism into $R$. Width 1 problems were characterized by the existence of a set function by Dalmau and Pearson in [3].

In the present paper we shall investigate the complexity of $\operatorname{EXT}(P)$ for a finite poset $P$. It turns out that there is a strong correlation between the complexity of the extendibility problem and the set of the monotone idempotent operations of the poset.

Algebras whose term operations coincide with the monotone operations of a poset are called order primal. Idempotent Malcev conditions satisfied in order primal algebras received much attention in the last decade. Some special operations play an important role in these investigations. Let $f$ be an $n$-ary operation. We say that $f$ is symmetric (cyclic) if it obeys the identity

$$
f\left(x_{1}, \ldots, x_{n}\right)=f\left(x_{\pi(1)}, \ldots, x_{\pi(n)}\right)
$$

for every permutation (for an $n$-cycle) $\pi$ of $\{1, \ldots, n\}$. The operation $f$ is called totally symmetric if it obeys the identity

$$
f\left(x_{1}, \ldots, x_{n}\right)=f\left(y_{1}, \ldots, y_{n}\right)
$$

for all sets of variables such that $\left\{x_{1}, \ldots, x_{n}\right\}=\left\{y_{1}, \ldots, y_{n}\right\}$. An $n$-ary operation $f$ is idempotent if it obeys the identity

$$
f(x, x, \ldots, x)=x .
$$


Let $n \geq 3$. An $n$-ary operation $f$ is called a near unanimity operation if the following $n$ identities hold:

$$
f(x, \ldots, x, \underbrace{y}_{i}, x, \ldots, x)=x, \quad i \in\{1, \ldots, n\} .
$$

The main results on special idempotent term operations in order primal algebras that we rely on are contained in [12] and [16]. In [12] the authors characterized the finite posets admitting a near unanimity operation. In [16] Szabó and Zádori gave a characterization of finite posets admitting a totally symmetric idempotent (TSI) operation of arity equal to the size of the poset. We note that the latter characterization can be derived from results on width 1 in [6] and [3].

In the present paper we investigate the relationship between the above-mentioned complexity and algebraic results. We show that if a poset $P$ admits a near unanimity operation, then it admits a TSI operation of arity $|P|$. This means that bounded strict width implies width 1 for extendibility problems of posets. We give an example showing that a similar implication does not hold for CS problems of relational structures. Further, we prove that $\operatorname{EXT}(P)$ is polynomial-time equivalent to a CS problem. Then by invoking Jeavons's ideas in [9] we generalize Pratt and Tiuryn's NP-completeness result on crowns.

2. Results. First we describe the relationship between two classes of extendibility problems for finite posets. Both of these classes lie in $\mathbf{P}$ and are relativized versions of important classes of CS problems.

We call a pair $(Q, f)$ a $P$-colored poset if $Q$ is a poset and $f$ is a partial map from $Q$ to $P$. The $P$-colored poset $(Q, f)$ or the partial map $f$ is called $P$-extendible if $f$ extends to a monotone total map from $Q$ to $P$. So we might consider $\operatorname{EXT}(P)$ to be the problem whose inputs are just the finite $P$-colored posets, and the task is to decide the extendibility of a $P$-colored poset.

Let $(Q, f)$ and $(H, g)$ be two $P$-colored posets. A monotone map $\alpha$ from $Q$ to $H$ is called a homomorphism from $(Q, f)$ to $(H, g)$ if $f=g \alpha$. The colored poset $(H, g)$ is a homomorphic image of $(Q, f)$ under the homomorphism $\alpha$ if $\alpha$ is onto. Now, $\operatorname{EXT}(P)$ might be considered to be the problem whose input is a finite $P$-colored poset $(H, g)$, and the task is to decide if there is a homomorphism from $(H, g)$ to the $P$-colored poset $\left(P, I d_{P}\right)$, where $I d_{P}$ is the identity map on $P$. Since in a CS problem the task also is to decide if there exists a homomorphism to a designated structure from a similar structure, the notions and theorems related to the CS problems in [6] easily transfer to extendibility problems of posets.

Note that there is a subtle difference that prevents us from considering $\operatorname{EXT}(P)$ to be a CS problem, namely that the instances of $\operatorname{EXT}(P)$ are certain poset structures while an instance for a CS problem is allowed to be any relational structure. Later in Proposition 9, we shall see that up to polynomial-time equivalence one can safely drop this restriction. For the time being we insist on this distinction.

Let $P$ be a finite poset. For $\operatorname{EXT}(P)$ the notions of width and strict width are defined in the same manner as those for CS problems in [6] of Feder and Vardi. By transferring the proofs of Theorems 19, 20, 22, and 24 in [6] and Theorem 1 in [3], one gets that $\operatorname{EXT}(P)$ has width 1 if and only if $P$ admits a TSI operation of arity $|P|$ and that $\operatorname{EXT}(P)$ has bounded strict width if and only if $P$ admits a near unanimity operation.

Let $H$ and $Q$ be finite posets. We say that $Q$ contains $H$ if the base set of $Q$ contains the base set of $H$ and the order relation of $Q$ contains the order relation of 
$H$. A finite $P$-colored poset $(Q, f)$ is called a zigzag if it is nonextendible but for any $H$ properly contained in $Q$ the $P$-colored poset $\left(H, f \uparrow_{H}\right)$ is extendible. A $P$-colored poset $(Q, f)$ is a tree if the covering graph of $Q$ is a tree. Let $(Q, f)$ and $(H, g)$ be two $P$-colored posets. For a finite poset $P$ let $I(P)$ be the algebra whose base set is the base set of $P$ and whose basic operations are the monotone idempotent operations of $P$. A subalgebra of a finite power of $I(P)$ is called an idempotent $P$-subalgebra. Note that the idempotent $P$-subalgebras inherit an order structure from $P$. Hence we shall consider them as posets. The notions defined in this paragraph play an important role in characterizations of finite posets that admit a near unanimity operation or a TSI operation of arity equal to the size of the base set of the poset.

TheOREM 1 (see [12]). For a finite connected poset $P$ the following are equivalent:

(1) $P$ admits a near unanimity operation.

(2) $P$ has finitely many P-zigzags.

(3) The idempotent P-subalgebras are dismantlable.

It is easy to prove (see [16]) that a finite poset $P$ admits a TSI operation of arity $|P|$ if and only if $P$ admits an $n$-ary TSI operation for all $n$.

TheOREM 2 (see [16]). A finite poset $P$ admits a TSI operation of arity $|P|$ if and only if every $P$-zigzag is a homomorphic image of a nonextendible tree.

Now, we are ready to prove that bounded strict width implies width 1 in the class of the extendibility problems for finite posets. By our earlier note it suffices to prove the following.

THEOREM 3. Every finite poset $P$ which admits a near unanimity operation also admits a TSI operation of arity $|P|$.

Proof. We refer to the proof of Theorem 4 in [16]. In the proof it is shown that if $P$ has finitely many zigzags and admits a cyclic operation of arbitrary arity, then every $P$-zigzag is a homomorphic image of a nonextendible tree. Now, we sharpen this result by showing that if $P$ has finitely many zigzags, then $P$ admits a cyclic idempotent operation of any arity. Then by Theorems 1 and 2 we get the claim for finite connected posets. For the case when $P$ is not connected and admits a near unanimity operation observe that the components of $P$ also admit a near unanimity operation. Moreover, the $P$-zigzags are the zigzags of the components and zigzags whose base posets are fences. Hence, the nonconnected case follows from the connected one.

So for the proof of this theorem let us suppose that $P$ has finitely many $P$ zigzags. Then by Theorem 1 every idempotent $P$-subalgebra is dismantlable. By a result of Rival in [14], every dismantlable poset has the fixed-point property. We apply this to the $P$-subalgebra $I_{n}$ formed by the $n$-ary monotone idempotent operations of $P$, $n=2,3, \ldots$ We define the monotone operation $\alpha_{n}$ on $I_{n}$ by

$$
\alpha_{n}: f\left(x_{1}, \ldots, x_{n}\right) \mapsto f\left(x_{2}, \ldots, x_{n}, x_{1}\right) .
$$

Since for each $n, I_{n}$ has the fixed point property, $\alpha_{n}$ has a fixed point. This fixed point is an $n$-ary cyclic idempotent operation for each $n$.

We remark that there exist finite relational sets of finite type which admit a near unanimity operation but no TSI operation of any arity. For example, let us consider a finite set $A$ with at least two elements and a ternary near unanimity operation $m$ on it. Let $R$ be the relational set whose base set is $A$ and whose relations are all binary relations preserved by $m$. It is well known in clone theory that, since $m$ is near unanimity, if $R$ admits an operation $f$, then $f$ is built from $m$ and the projections via composition. But since $m$ is a near unanimity operation, the only binary operations obtained in this way are projections. Hence $R$ admits no binary TSI operation and hence none of any higher arity either. 
There are simple examples of posets which admit a TSI operation of every arity but no near unanimity operation, for example, the poset $2+2+1$; see [16]. We do not know if bounded width implies width 1 in the class of the extendibility problems of finite posets.

During the editorial process of this paper Kun and Szabó have published paper [10] in which they describe a polynomial-time algorithm that decides if a finite poset admits a near unanimity operation. This improves the decidability result in [12]. In comparison, no algorithm is known for finite structures of finite type to decide if they admit a near unanimity operation. In the same paper Szabó and Kun actually construct a symmetric idempotent operation of any arity for any poset that admits a near unanimity operation.

Next we shall prove that for every poset which admits no nontrivial idempotent Malcev condition, the extendibility problem is NP-complete. It was observed by Corominas in [2] that the only idempotent monotone operations on crowns are the projections. Hence crowns admit no nontrivial idempotent Malcev condition and Pratt and Tiuryn's result on crowns is a consequence of ours. Our proof will be based on a polynomial-time reduction of $\operatorname{EXT}(P)$ to a CS problem. Once this reduction is established, the NP-completeness of $\operatorname{EXT}(P)$ follows from results in [1] and [9].

Let $M$ be a finite set of identities in the first order language of some algebra $A$. The set $M$ is called trivial if there is a two element algebra similar to $A$ whose basic operations are projections satisfying $M$. The set $M$ is called idempotent if in any algebra the term operations satisfying $M$ are idempotent. We say that an algebra $B$ admits a nontrivial Malcev condition if there is a nontrivial set of identities satisfied by some term operations of $B$. We call an algebra trivial if its basic operations are projections. Let $n \geq 2$. An $n$-ary idempotent operation $f$ is called a Taylor operation if it satisfies $n$ identities of the form

$$
f(\ldots, \underbrace{x}_{i}, \ldots)=f(\ldots, \underbrace{y}_{i}, \ldots), i=1,2, \ldots, n,
$$

where $x$ and $y$ are the only variables occurring in the identities and $x \neq y$. The proof of the following theorem is due to Taylor; see Corollaries 5.2 and 5.3 in [17]. An alternate proof is given in [8]; see Theorem 9.4.

Theorem 4 (see [17]). Let $A$ be a idempotent algebra. Then the following are equivalent:

(1) A admits no nontrivial idempotent Malcev condition.

(2) There is a two element trivial algebra in the variety generated by A.

(3) There is no Taylor operation among the term operations of $A$.

If $A$ is a finite idempotent algebra satisfying the equivalent conditions of the preceding theorem, then a two element trivial algebra is a homomorphic image of a subalgebra of $A^{n}$ for some finite $n$. In fact, more is true.

PROPOSITION 5. Let $B$ be a two element algebra which is a homomorphic image of a subalgebra of a finite power of an idempotent algebra $A$. Then $B$ is a homomorphic image of a subalgebra of $A$.

Proof. Let $C$ be a subalgebra of $A^{n}$, let $h$ be a homomorphism of $C$ onto $B$, and assume that $n>1$ is the least integer for which this situation holds. Let $\pi_{i}$ denote the $i$ th projection of $A^{n}$ onto $A$, and let $f_{i}$ denote the restriction of $\pi_{i}$ to $C$. Let $D$ denote the image of $f_{i}$. Suppose first that the kernel of $f_{i}$ is contained in the kernel of $h$. Then by the second isomorphism theorem there exists a homomorphism of $D$ onto $B$ and this contradicts our choice of $n$. Hence there exists a pair $(a, b)$ of elements of $C$ such that $h(\{a, b\})=B$ and $a_{i}=b_{i}=c$ for some $c \in A$. However, since $A$ is an 
idempotent algebra, the set $X$ of all $x \in A^{n}$ such that $x_{i}=c$ is a subalgebra of $A^{n}$; in fact, it can clearly be embedded in $A^{n-1}$ and it admits a homomorphism onto $B$, contradicting once more our choice of $n$.

Let $A$ be an algebra and $R$ a relational set on the same base set. We say that $A$ is an algebra for $R$ or $R$ is a relational set for $A$ if the set of term operations of $A$ coincides with the set of morphisms from finite powers of $R$ to $R$. The following result is due to Jeavons; see Corollary 4.11 in [9].

TheOrem 6 (see [9]). Let $Q$ and $R$ be finite relational sets on the same base set and let $A$ be an algebra for $Q$. If $R$ is of finite type and all the relations of $R$ are subalgebras of finite powers of $A$, then $\operatorname{CSP}(R)$ reduces to $C S P(Q)$ in polynomial time.

The proof of the following result is basically contained in [1].

TheOrem 7. Let $R$ and $Q$ be finite relational sets where $R$ is of finite type. Suppose that $A$ is an algebra on the same base set as $R$ such that the relations of $R$ are subalgebras of finite powers of $A$ and that $B$ is an algebra for $Q$. If $A$ is a homomorphic image of a subalgebra of $B$, then there is a polynomial-time reduction of $\operatorname{CSP}(R)$ to $\operatorname{CSP}(Q)$.

Proof. Let $C$ be a subalgebra of $B$ and $\varphi$ a homomorphism from $C$ onto $A$. Let $R^{\prime}$ be the relational set whose base set is the base set of $Q$ and whose relations are the inverse images of the relations of $R$ under $\varphi^{-1}$ together with the unary relation $C$. It is a routine exercise to show that the relations of $R^{\prime}$ are preserved under the operations of $B$. So, it follows that the relations of $R^{\prime}$ are subalgebras of finite powers of $B$. Now, any instance $X$ of $\operatorname{CSP}(R)$ is made into an instance $Y$ of $\operatorname{CSP}\left(R^{\prime}\right)$ by adding the base set of $X$ as the unary relation corresponding to $C$. Moreover, $X$ is a satisfying instance of $\operatorname{CSP}(R)$ if and only if $Y$ is a satisfying instance of $\operatorname{CSP}\left(R^{\prime}\right)$. So $\operatorname{CSP}(R)$ reduces to $\operatorname{CSP}\left(R^{\prime}\right)$ in polynomial time. By the preceding theorem $\operatorname{CSP}\left(R^{\prime}\right)$ reduces to $\operatorname{CSP}(Q)$ in polynomial time, which concludes the proof.

By a result of Schaefer [15], there exists a relational set $R$ of finite type on the two element set such that $\operatorname{CSP}(R)$ is $\mathbf{N P}$-complete. Combining this fact with the preceding theorems we get the following.

Corollary 8. Let $A$ be an idempotent algebra for a finite relational set $Q$. If $A$ admits no nontrivial idempotent Malcev condition, then $C S P(Q)$ is NP-complete.

Proof. Let $R$ be a relational set of finite type on two elements such that $\operatorname{CSP}(R)$ is NP-complete. By Theorem 4 and Proposition 5, there exists a trivial two element algebra $B$ which is a homomorphic image of a subalgebra of $A$. Since $B$ is trivial, the relations of $R$ are subalgebras of finite powers of $B$. Hence by Theorem 7 there is a polynomial-time reduction of $\operatorname{CSP}(R)$ to $\operatorname{CSP}(Q)$.

We say that a poset $P$ admits a nontrivial idempotent Malcev condition if $I(P)$ admits a nontrivial idempotent Malcev condition. Posets admitting a TSI operation are examples of such posets since any TSI operation is a Taylor operation. Next we show that if $P$ admits no nontrivial Malcev condition, then $\operatorname{EXT}(P)$ is NP-complete. Let $P_{P}$ be the relational set defined by equipping the base set of $P$ with the order relation of $P$ and all constants of $P$ as one element unary relations. Clearly, $P_{P}$ is a relational set for $I(P)$.

Proposition 9. The problems EXT $(P)$ and $C S P\left(P_{P}\right)$ are polynomial-time equivalent.

Proof. First we reduce $\operatorname{EXT}(P)$ to $\operatorname{CSP}\left(P_{P}\right)$ in polynomial time. Let $(\mathrm{Q}, \mathrm{f})$ be an instance for $\operatorname{EXT}(P)$. By adding the unary relation $f^{-1}(p)$ for each $p \in P$ to the poset $Q$, we define an instance $Q_{P}$ of $\operatorname{CSP}\left(P_{P}\right)$. Note that $(\mathrm{Q}, \mathrm{f})$ is extendible if and 
only if there is a morphism from $Q_{P}$ to $P_{P}$.

Next we reduce $\operatorname{CSP}\left(P_{P}\right)$ to $\operatorname{EXT}(P)$ in polynomial time. Since $P_{P}$ is of finite type we can assume without loss of generality that the instances of $\operatorname{CSP}\left(P_{P}\right)$ are relational sets similar to $P_{P}$. So let $X$ be an arbitrary relational set similar to $P_{P}$. The reflexive, transitive closure of the binary relation of $X$ is a quasi order, say $\alpha$, on $P$. Let $\theta=\alpha \cap \alpha^{-1}$, the equivalence determined by $\alpha$, as usual. Let $\bar{x}$ denote the $\theta$-block of $x$ for each $x \in X$. We associate a $P$-colored poset $(H, f)$ with $X$, where $H=X / \theta$ and for each $x \in X, f(\bar{x})=p$ if and only if $x$ is in the unary relation of $\mathrm{X}$ that corresponds to $p$. So $f(\bar{x})$ is not defined whenever no unary relation of $X$ contains $x$. In case that $f$ is not well defined, $X$ is not a satisfying instance of $\operatorname{CSP}\left(P_{P}\right)$. So we restrict ourselves to the case when $f$ is well defined. Observe that the kernel of any morphism from $X$ to $P_{P}$ contains $\theta$. So $X$ is a satisfying instance of $\operatorname{CSP}\left(P_{P}\right)$ if and only if $(H, f)$ is extendible.

Corollary 10. If $P$ is a finite poset which admits no nontrivial Malcev condition, then $\operatorname{EXT}(P)$ is $\mathbf{N P}$-complete.

Proof. By Proposition 9 it suffices to show that $\operatorname{CSP}\left(P_{P}\right)$ is NP-complete. Now, $I(P)$ is an idempotent algebra for $P_{P}$ such that $I(P)$ admits no nontrivial idempotent Malcev condition. Then by Corollary 8 the problem $\operatorname{CSP}\left(P_{P}\right)$ is NP-complete.

Posets admitting only projections as idempotent operations have been studied in numerous papers, e.g., [2], [4], [7], and [11]. Any poset $P$ with an idempotent $P$-subalgebra that retracts onto one of these posets admits no nontrivial Malcev condition and hence yields an example where $\operatorname{EXT}(P)$ is NP-complete.

3. Concluding remarks. We saw that for the finite posets admitting a TSI operation of large enough arity the extendibility problem has polynomial-time complexity. On the other hand, we obtained that for finite posets which admit no nontrivial idempotent Malcev condition the extendibility problem is NP-complete.

Are there any finite posets not included in the two classes of posets mentioned in the preceding paragraph? There are CS problems without bounded width in $\mathbf{P}$ which are polynomial-time equivalent to the extendibility problem for a poset as proven in [6]. The anonymous referee, whom we thank for his/her judicious advice, noted that a resulting poset of that type might not have bounded width either. Nonetheless, the question still remains open.

Neither are we able to answer the following simpler question. Is there any finite poset $P$ that admits a binary idempotent commutative operation but does not admit a TSI operation of arity $|P|$ ?

Acknowledgment. The authors would like to thank Pavol Hell for patiently mediating between the referee of the paper and the authors.

\section{REFERENCES}

[1] A. A. Bulatov, P. G. Jeavons, and A. A. Krokhin, Constraint satisfaction problems and finite algebras, in Proceedings of the 27th International Colloquium on Automata, Languages and Programming, ICALP'00, Lecture Notes in Comput. Sci. 1853, Springer-Verlag, Berlin, 2000, pp. 272-282.

[2] E. CoRominas, Sur les ensembles ordonnés projectif et la properiétré du point fixe, C. R. Acad. Sci. Paris Sér. I Math., 311 (1990), pp. 199-204.

[3] V. Dalmau and J. Pearson, Closure functions and width 1 problems, in Proceedings of the 5th International Conference on Principles and Practice of Constraint Programming, CP'99, Lecture Notes in Comput. Sci. 1713, Springer-Verlag, Berlin, 1999, pp. 159-173.

[4] B. A. Davey, J. B. Nation, R. N. McKenzie, and P. P. PÁlfy, Braids and their monotone clones, Algebra Universalis, 32 (1994), pp. 153-176. 
[5] D. Duffus and T. Goddard, The complexity of the fixed point property, Order, 13 (1996), pp. 209-218.

[6] T. FEDER AND M. Y. VARDI, The computational structure of monotone monadic SNP and constraint satisfaction: A study through datalog and group theory, SIAM J. Comput., 28 (1998), pp. 57-104.

[7] S. Hazan, Two properties of projective orders, Order, 9 (1992), pp. 233-238.

[8] D. Hobby and R. McKenzie, The Structure of Finite Algebras, Contemp. Math. 76, AMS, Providence, RI, 1988.

[9] P. Jeavons, On the algebraic structure of combinatorial problems, Theoret. Comput. Sci., 200 (1998), pp. 185-204.

[10] G. Kun and Cs. Szabó, Order varieties and monotone retractions of finite posets, Order, 18 (2001), pp. 79-88.

[11] B. LARose, Minimal automorphic posets and the projection property, Internat. J. Algebra Comput., 5 (1995), pp. 65-80.

[12] B. Larose And L. ZÁdori, Algebraic properties and dismantlability of finite posets, Discrete Math., 163 (1997), pp. 89-99.

[13] V. Pratt and J. Tiuryn, Satisfiability of inequalities in a poset, Fund. Inform., 28 (1996), pp. $165-182$.

[14] I. RIVAL, A fixed point theorem for finite partially ordered sets, J. Combin. Theory Ser. A, 21 (1976), pp. 309-318.

[15] T. J. SchaEfer, The complexity of the satisfiability problems, in Proceedings of the 10th ACM Symposium on the Theory of Computing (STOC), 1978, pp. 216-226.

[16] Cs. Szabó And L. ZÁdori, Idempotent totally symmetric operations on finite posets, Order, 18 (2001), pp. 39-47.

[17] W. TAYLOR, Varieties obeying homotopy laws, Canad. J. Math., 29 (1977), pp. 498-527. 